\title{
La variabilidad dimensional y defectos de secado afectan rendimientos en procesos de cepillado de madera de Eucalyptus saligna
}

\author{
Dimensional variability and drying defects affect yields during brushing \\ processes of Eucalyptus saligna wood
}

\author{
Napoleón López ${ }^{\text {a*, }}$ María Fernanda Yucta ${ }^{\text {b }}$, Karina Caraguay ${ }^{\text {b }}$, Rumiñahui Minga ${ }^{\text {b }}$ \\ *Autor de Correspondencia: ${ }^{\text {a } U n i v e r s i d a d ~ N a c i o n a l ~ d e ~ L o j a, ~ C e n t r o ~ d e ~ l a ~ M a d e r a, ~}$ \\ Ciudadela Universitaria “Guillermo Falconí Espinoza”, La Argelia, Loja, Ecuador, tel.: 0999681876, napoleon.lopez@unl.edu.ec \\ ${ }^{\mathrm{b}}$ Universidad Nacional de Loja, Área Agropecuaria y de los Recursos Naturales Renovables, \\ Carrera de Ingeniería Forestal, Loja, Ecuador.
}

\begin{abstract}
SUMMARY
The losses due to the large number of defects of introduced wood Eucalyptus saligna during air drying were investigated in order to improve utilization yield and quality of wood planing processes. The objective was to evaluate dimensions and defects in 300 commercial pieces: boards $(240 \times 12 \times 3 \mathrm{~cm})$ and 300 tables $(300 \times 20 \times 2 \mathrm{~cm})$, which were subjected to a process of drying on the open air for more than a year. The volume of clean wood slats was obtained for average and $8,139 \mathrm{~cm}^{3}$ were reduced to $2,243 \mathrm{~cm}^{3}$, after a theoretical defect removal, ie hypothetically discounted the geometric volume of each defect. This represented a $27.5 \%$ yield of clean wood and a loss of $73.4 \%$ by defect removal. In tables, the average volume was $13.240 \mathrm{~cm}^{3}$ and the clean volume was calculated $4,406 \mathrm{~cm}^{3}$, an amount that represented $33.2 \%$ least additively to calculate correction. Given this, the removal defect by brushing and squareness was processed in a sample of 15 pieces; this reached yields of $58.4 \%$ for tablets and $51.8 \%$ for tables, which meant losses of 41.6 and $48.2 \%$ respectively; these losses in the brushing process were investigated showing that they affect the performance of E. saligna.
\end{abstract}

Key words: harvesting, eucalyptus, performance, workability.

\section{RESUMEN}

Las pérdidas debido a la gran cantidad de defectos que presentó la madera de Eucalyptus saligna, durante el proceso de secado al aire libre, fueron investigadas con el objeto de mejorar rendimientos de aprovechamiento y la calidad de madera en procesos de cepillado. El trabajo consistió en evaluar dimensiones y defectos en 300 piezas comerciales: tablillas $(240 \times 12 \times 3 \mathrm{~cm})$ y 300 tablas $(300 \times 20 \times$ $2 \mathrm{~cm}$ ), que estuvieron sometidas a un proceso de secado al aire libre por más de un año. El volumen de madera limpia obtenido para tablillas fue de $8.139 \mathrm{~cm}^{3}$ promedio y fue reducido a $2.243 \mathrm{~cm}^{3}$, luego de una eliminación teórica de defectos, es decir descontado hipotéticamente el volumen geométrico de cada defecto; esto representó un $27,5 \%$ de rendimiento en madera limpia y una pérdida de $73,4 \%$ por eliminación de defectos. En tablas el volumen medio fue de $13.240 \mathrm{~cm}^{3}$ y el volumen limpio calculado fue de $4.406 \mathrm{~cm}^{3}$, cantidad que representó un 33,2 \% menos, al calcular de forma aditiva su corrección. Ante esto se procesó la eliminación de defectos por cepillado y escuadría en una muestra de 15 piezas y se alcanzó rendimientos de 58,4 \% para tablillas y 51,8 \% para tablas, lo que significó pérdidas de 41,6 y 48,2\%, respectivamente; estas pérdidas en el proceso de cepillado fueron investigadas demostrándose que afectan el rendimiento del E. saligna.

Palabras clave: aprovechamiento, eucalipto, rendimiento, trabajabilidad.

\section{INTRODUCCIÓN}

Los estudios de trabajabilidad en el género Eucalyptus van en aumento año a año, la producción de madera aserrada es una realidad en Brasil (Del Menezzi y Sousa 2001), los precios de la madera aserrada superan cuatro veces al precio de la madera para celulosa de fibra, señala De Freitas (2008), por tanto ayudar a mejorar procesos que se adapten a las propiedades de la madera de eucalipto para ofrecer beneficios mediante reducción de pérdidas en rendimiento y menores costos de producción, es una problemática que requiere de mayor investigación. Si bien los procesos tecnológicos de aserrado evolucionan día a día, en capacidad productiva, eficiencia y comodidad o facilidad operativa, la tecnología del secado no responde en igual forma, sobre todo para Eucalyptus spp., una gran parte de la materia prima procesada se pierde por: variabilidad dimensional del aserrado, contracción característica anisotrópica propia de las maderas y defectos en el proceso de secado, lo que afecta el rendimiento y calidad de la 
madera al momento de ser utilizada, razón por la que se conduce esta investigación en piezas comerciales. Como manifiesta De Freitas et al. (2008), una de las alternativas más inmediatas para suplir la demanda de madera es el desarrollo de técnicas de procesamiento para el mercado de eucalipto

Bajo este análisis se planteó como objetivo de investigación evaluar la variabilidad dimensional y los defectos en dos tipos de piezas de madera aserrada de Eucalyptus saligna Smith y cuantificar rendimientos en procesos de cepillado. Definiendo como hipótesis que la variabilidad dimensional y defectos afectan el rendimiento de la madera cepillada del E. saligna.

En la provincia de Loja sur del Ecuador, el E. salig$n a$ tiene buen crecimiento y pese a sus características la madera no es bien aprovechada por los problemas en aserrado y posteriormente en secado, calificada por algunos carpinteros o artesanos como una madera que no vale para trabajos de carpintería. Ante este desconocimiento tecnológico, hay que hacer algo para solucionar el problema de reforestadores y pequeños artesanos, que puedan hacer un mejor uso y valorar los bosques en condiciones de ser aprovechados y a la vez abrir la perspectiva a futuro de seguir plantando esta especie que tiene un potencial en cuanto a su crecimiento.

Loja y el país requieren orientar las actividades de reforestación productiva principalmente a fin de sustituir la madera proveniente de bosques nativos, mejorar los rendimientos y disminuir costos de producción, incorporar procesos tecnológicos que ayuden a generar fuentes de trabajo, una de estas actividades que requiere atención inmediata es poder controlar los defectos que se producen en aserrado como en secado de la madera de E. saligna para mejorar su trabajabilidad dar buen uso a las plantaciones establecidas y que demuestran una buena adaptabilidad y crecimiento en la provincia de Loja.

Eucalyptus saligna surge como una especie alternativa para productos sólidos, en especial, aquellos con valor agregado. En ciertos casos puede sustituir a maderas nativas tropicales, lo que le hace una especie interesante ${ }^{1}$. Es un árbol de gran tamaño, que puede alcanzar de $40 \mathrm{a}$ $55 \mathrm{~m}$ de altura y de 1,2 a $2,0 \mathrm{~m}$ de diámetro a la altura del pecho. Árboles excepcionales llegan a los $65 \mathrm{~m}$ de alto y a los 2,5 $\mathrm{m}$ de diámetro. Tiene muy buena forma con fuste recto, libre de ramas hasta la mitad o las dos terceras partes de la altura total del árbol (CATIE 1991). Esta especie es originaria de Quesland y Nueva Gales del Sur en Australia, reconocida así por más de 100 años en la misma Australia, pero en 1918, Maiden establece una nueva especie, $E u$ calyptus grandis Hill (Maiden) (FAO 1981). En Ecuador no se encuentran registros de su procedencia y no se tiene

Piter JC, E Torran, I Villalba, C Calvo, A Cuffre, C Tejedor, A Sosa Zitto, N Zakowicz, M Sánchez Acosta. 2002. Contractibilidad de la madera de Eucalyptus grandis de distintas poblaciones genéticas de la Mesopotamia. Grupo GEMA - Universidad Tecnológica Nacional UTN. Concordia, Entre Ríos, Argentina. (No publicado). la certeza sobre la especie, pero comúnmente se habla de E. saligna (López 2001).

La madera tiene un color rosado, aunque también tiende a blanquecino, en ensayos comparativos de E. saligna y E. grandis de 5 años de edad en Hawai, (Skolmen 1986), se obtienen mejores crecimientos en E. grandis con una densidad media de $0,367 \mathrm{~g} \mathrm{~cm}^{-3}$, sin embargo, la densidad específica de la madera es mayor para el E. saligna $0,413 \mathrm{~g} \mathrm{~cm}^{-3}, 15$ años más tarde plantaciones de E. saligna hawaianas registraron densidades entre 0,349 y $0,496 \mathrm{~g}$ $\mathrm{cm}^{-3}$ (DeBell et al. 2001); en otros estudios sobre densidad de E. saligna, López (2001) obtiene $0,49 \mathrm{~g} \mathrm{~cm}^{-3}$, contracción total del 10,4 \% al 8,7\% de contenido de humedad, Da Silva y Castro (2003), en densidad para E. saligna obtienen $0,47 \mathrm{~g} \mathrm{~cm}^{-3} \mathrm{y}$ contracción volumétrica del $26 \%$ y un factor anisotrópico de 1,99.

El control de calidad es vital para la industria maderera, de ahí la necesidad de llevar un control del producto que se elabora. La calidad de la madera aserrada puede ser evaluada por sus características naturales como las propiedades físicas y por la precisión de sus dimensiones. La variación dimensional de las tablas aserradas, síntoma de baja calidad, dificulta la comercialización y en consecuencia, la baja competitividad de la industria del aserrío (Nájera et al. 2006). Al mismo tiempo, la variación del aserrío influye significativamente en el rendimiento y calidad de la madera.

La variación dimensional de las tablas aserradas influye significativamente en el rendimiento y es el grueso o espesor de asierre la dimensión más crítica de controlar, por lo que es importante utilizar equipos sin vibraciones, sierras en buen estado y tener otras consideraciones de control. La combinación de albura y duramen en una misma tabla induce a un comportamiento diferenciado en la contracción durante el secado. Sus efectos son mayores en la porción de albura donde la contracción es más severa que en el duramen. De la misma manera, las tablas tangenciales son más susceptibles a presentar rajaduras superficiales y acanalamientos durante el secado, defectos que impactan directamente sobre la calidad de la madera aserrada (Nájera et al. 2006).

Independientemente a la variabilidad dimensional por aserrado las maderas presentan la contracción natural de la madera que puede ser el doble en el corte tangencial en relación al corte radial y es menor en corte longitudinal, los valores del mencionado cociente de contracción (T/R) conocido como coeficiente de anisotropía, comprendidos entre 1,2 y 1,5 pueden considerarse excelentes, en tanto que entre 1,6 y 1,9 se destacan como normales y los mayores a 2,0 originan dificultades para determinados usos de la madera (Calvo et al. 2006).

Durante el secado se presenta una serie de defectos como rajaduras y alabeos sobre las que debe incorporarse recomendaciones técnicas como: nivelado, sombreado, presión o carga, separadores y otras para mejorar la calidad de madera de eucalipto (De Freitas 2008). Los defec- 
tos como las rajaduras no son producidos únicamente por secado, las rajaduras en extremos de rollizos y tablas se producen por la liberación de las tensiones de crecimiento. Dichas tensiones, según citan López y Perisi (2002), se generan durante el crecimiento y maduración de las células producidas por el cambium. En general, mientras el árbol está en pie, las tensiones mantienen un equilibrio a lo largo del tronco, inmediatamente después del apeo, dichas tensiones comienzan a liberarse y este reordenamiento origina, en primera instancia, rajaduras en los extremos de los rollizos y, posteriormente, rajaduras y diversos tipos de alabeos en las piezas aserradas.

Sánchez Acosta (2006), al referirse a la experiencia en secado de eucaliptos, señala que para atenuar los problemas se hace indispensable utilizar las tecnologías apropiadas en la industrialización como: tipos de corte de aserrado, secado lento y temperaturas adecuadas, entre otros.

Martins (2009), en comparación de secado al aire libre y secado solar para madera de E. saligna señala que un buen apilado minimiza los defectos de encorvadura, no presenta defectos de acanalado, se presenta mayores defectos de arqueaduras en secado solar, en cambio las rajaduras mayormente en secado al aire libre; ello justifica un secado al aire modificado bajo techo, que proteja la lluvia como los rayos solares.

La presencia de grietas asociadas con problemas de contracción durante el secado exhiben una gran variedad en los eucaliptos, siendo Eucalyptus grandis la especie que en general presenta menores problemas (Hillis 1978, INTA 1995). No obstante, se reportan deformaciones y grietas que pueden limitar la utilización de esta especie cultivada en Argentina ${ }^{2}$.

Generalmente se realizan estudios en cuanto a rendimientos de transformación de madera aserrada, así De Freitas (2008), obtiene $26 \%$ y $24 \%$ de rendimiento desde trozas de E. saligna y en madera seca al horno con $14 \%$ de contenido de humedad respectivamente; ante esto se requiere de mayores estudios de secado que encierren otras variables como apilado, circulación del aire, disposición de piezas, entre otras.

Sin embargo, no se encuentra estudios, que permitan reflejar las verdaderas pérdidas de madera por defectos que se presentan en secado y su posterior aprovechamiento, que es donde está el problema para el eucalipto, porque la pieza aún con defectos aceptables desde el punto de vista comercial tiene que ser procesada y escuadrada, hay que eliminar los defectos mediante un cepillado.

Por tanto, la hipótesis planteada en este trabajo es que la variabilidad dimensional y defectos del secado afectan el rendimiento de la madera de Eucalyptus saligna, con lo cual se pretende demostrar objetivamente que hay pérdida importante de madera al ser cepillada.

\footnotetext{
Ver nota 1
}

\section{MÉTODOS}

En la ciudad de Catamayo, provincia de Loja-Ecuador, se aprovechó de la existencia de madera de Eucalyptus saligna apilada bajo techo por más de un año, en donde por las condiciones climáticas la madera alcanzó un promedio de $9,4 \%$ de contenido de humedad, se seleccionaron 300 tablillas de $240 \times 12 \times 3 \mathrm{~cm}$ y 300 tablas de $300 \times 20 \times 2 \mathrm{~cm}$, que fueron sometidas a un proceso de medición dimensional, evaluación de defectos y luego en una muestra de 15 piezas de tablillas y 15 tablas se realizó un proceso de cepillado y escuadrado de las piezas para eliminar defectos, lo que permitió obtener el volumen antes y después para cálculos de rendimiento.

Evaluación dimensional. Cada pieza se codificó y se marcaron tres puntos de medición en cada una, a un décimo de su longitud en cada extremo y al centro, esto es a $25 \mathrm{~cm}$ en tablillas y $30 \mathrm{~cm}$ en tablas en donde se midió con escuadra el ancho en ambas caras de la pieza con precisión de $1 \mathrm{~mm}$, el espesor se tomó con calibrador en los cantos a la señal marcada (seis puntos) y la longitud se midió con flexómetro en los dos cantos con precisión de $1 \mathrm{~mm}$, para luego por diferencia en extremos, centro y cantos analizar su variabilidad:

$$
\mathrm{V}=\mathrm{Dp} 1-\mathrm{Dp} 2
$$

Donde: $\mathrm{V}=$ variabilidad. DP1 = Dimensión en punto uno (espesor, cara o canto). Dp2 = Dimensión en punto dos (espesor, cara o canto).

Medición de defectos. Se evaluó los defectos de secado acanaladura, combado o arqueado, torcedura, encorvadura y rajadura en los extremos de cada pieza. Los defectos se determinaron de conformidad con las normas propuestas por la Junta del Acuerdo de Cartagena para países del grupo andino JUNAC (1984).

- Acanaladuras. La medición se determinó por la distancia mayor que existe entre la arista cóncava de la pieza y el plano transversal que une las esquinas o cantos. Adicionalmente se evalúo el lado convexo, es decir, la otra cara de la pieza, toda vez que en proceso de cepillado la pieza debe ser corregida por ambos lados. En otras palabras, el espesor de la madera aserrada menos el espesor de la madera cepillada, propuesto por De Freitas et al. (2008).

- Arqueaduras. Se determinó por la distancia mayor existente entre la cara y un plano horizontal que une los dos extremos de la pieza. Así mismo se determinó la curvatura saliente sobre el lado convexo de la cara de la pieza.

- Encorvaduras. Se midió la luz o distancia mayor entre el canto y el plano horizontal. Igualmente se determinó la curvatura sobresaliente en el lado convexo de la pieza, toda vez que la pieza debe ser escuadrada. 
- Torceduras. Se midió únicamente la distancia de la arista levantada sobre el plano en el cual se apoyan las aristas restantes.

- Rajaduras. Su determinación fue longitudinal en forma directa. Por ser uno de los problemas de la madera de eucalipto, se determinó la frecuencia para cada extremo y se medió la rajadura de mayor longitud en cada extremo.

Rendimiento. Cumplido el proceso de evaluación de 300 tablas y 300 tablillas, se tomó una muestra de 15 tablas y 15 tablillas, muestras que fueron sometidas a un proceso de cepillado de cantos y caras que permita corregir los defectos presentados dejando las piezas escuadradas, luego de lo cual una vez más se tomó las medidas finales, largo, ancho y espesor, para obtener el volumen de madera limpia o cepillada.

Con el promedio de dimensiones tomadas en cada pieza se calculó el volumen inicial y final de las piezas para aplicar la siguiente relación:

$$
\mathrm{R} \%=\left(\mathrm{Vcep} \mathrm{cm}^{3} / \text { Vase } \mathrm{cm}^{3}\right) \times 100
$$

Donde: $\mathrm{R}=$ Rendimiento en porcentaje. Vcep $=$ Volumen de piezas secas cepilladas, $\mathrm{cm}^{3}$.

Vase $=$ Volumen de piezas aserradas, $\mathrm{cm}^{3}$.

Tratamiento de la información. La variabilidad dimensional en ancho y espesor de la pieza a partir de los extremos y centro de cada pieza, y en los cantos para la longitud, permitieron obtener promedios de la variabilidad en las tres dimensiones de la pieza, los cuales se utilizaron en un análisis de grupos para las dimensiones de: longitud, ancho y espesor, haciendo uso del programa estadístico SPSS versión 19.

Para cada defecto de secado se midió en el lado cóncavo y convexo de la pieza, dimensión que se utilizó para calcular volumen de corrección según como se presentó el defecto y fueron considerados como si las pérdidas se eliminarán directamente en forma individualizadas, luego sus promedios se analizaron estadísticamente por grupos de comparación: acanaladuras, torceduras, arqueaduras.

El rendimiento limpio en base al volumen antes y después del cepillado y escuadrado de piezas, permitió demostrar que la variabilidad dimensional y defectos del secado afectan el rendimiento final luego del proceso de cepillado de la madera. La diferencia respecto al volumen inicial significa pérdidas de madera de eucalipto.

\section{RESULTADOS}

En la variabilidad dimensional [1] de la madera de $E$. saligna influyó el proceso de aserrado, además, el secado con la contracción propiedad natural de la madera y los defectos presentados debido a las condiciones de crecimiento, apeo, proceso de secado, apilado, entre otros.
La variabilidad dimensional longitudinal de la madera, en tablillas de $240 \mathrm{~cm}$ de longitud promedio, obtuvo una desviación estándar (sd) de $6 \mathrm{~mm}$ y para tablas de longitud media de 300,2 cm, obtuvo una desviación estándar de $9 \mathrm{~mm}$ y se presentó principalmente por falta de escuadría en los extremos, pero que no afectó un volumen considerable.

La variabilidad en ancho de tablillas de $11,4 \mathrm{~cm}$ promedio, alcanzó una desviación estándar de 3 y $5 \mathrm{~mm}$ en los extremos de las piezas y en las tablas de 19,6 cm de ancho promedio, la desviación estándar fue de $2 \mathrm{~mm}$ en los extremos y centro de la pieza.

La variabilidad dimensional en espesor para tablillas aserradas con $3,0 \mathrm{~cm}$ promedio, obtuvo una desviación estándar de $1 \mathrm{~mm}$ y para tablas con promedio de $2,3 \mathrm{~cm}$ de espesor, la desviación estándar fue $2 \mathrm{~mm}$.

La corrección de esta variabilidad dimensional y, sobre todo, la dimensión en espesor afectaron a toda la pieza e inclusive al lote de piezas porque se colocó en un solo espesor, el más bajo a fin de tener piezas estándar (cuadro 1).

La variabilidad mayor se presentó en espesor de tablas aserradas con motosierra, mientras que las tablillas se aserraron con sierra cinta; sin embargo, el análisis entre puntos medidos en extremos y centro de las piezas no mostró diferencia.

El defecto de arqueadura tuvo mayor magnitud en tablillas, pero fue más variable en tablas, posiblemente por flexibilidad en las piezas. El defecto de encorvadura, en cambio, fue mayor en tablas al igual que las torceduras y acanaladuras que están relacionadas al ancho de la pieza, como puede apreciarse en el cuadro 2 .

Los datos de cuantificación de estos defectos registrados en lado cóncavo y convexo de cada pieza, duplicaron la dimensión donde influye el defecto y fueron utilizados para obtener un volumen denominado de corrección. Equivale a la pérdida de madera para cada defecto, que convertida a porcentaje expresa la magnitud teórica de las pérdidas (cuadros 3 y 4 ).

Las rajaduras son defectos que, dependiendo de su posición, afectaron a la longitud de toda la pieza, además presentaron una amplia variabilidad y se registró $59,5 \mathrm{~cm}$ en promedio para tablillas y $63,7 \mathrm{~cm}$ para tablas.

Los defectos que presentó el Eucalyptus saligna, demostraron no ser aditivos ya que el mayor defecto enmascaró a los de menor magnitud. El defecto de arqueadura, que es mayoritario, no se corrigió de manera directa y por la flexibilidad de la madera aún permaneció luego del cepillado, razón que explica sobrepasar el porcentaje respecto de sus dimensiones.

Las acanaladuras y torceduras afectaron a ambas caras de la pieza y fueron las que ocasionaron mayor pérdida en tiempo y trabajo, debido a que su corrección significó varias pasadas de cepillado y dificultad al inicio del proceso porque la pieza quedó presionada en la máquina, necesitó de mayor fuerza o en su defecto liberar del roce de avance. La cuantificación de los defectos permite confirmar la 
hipótesis de que los defectos de secado ocasionan pérdidas de madera (figura 1); y a su vez, el rendimiento alcanzado luego de la corrección de defectos mediante cepillado, ratifica importantes pérdidas, sobretodo, para tablas (figura 2).

Cuadro 1. Variabilidad dimensional en madera aserrada de Eucalyptus saligna $(\mathrm{n}=300)$.

Variability dimensional lumber Eucalyptus saligna $(\mathrm{n}=300)$.

\begin{tabular}{llccc}
\hline \multicolumn{1}{c}{ Factor análisis } & \multicolumn{1}{c}{ Variable } & $\begin{array}{c}\text { Dimensión promedio } \\
(\mathrm{cm})\end{array}$ & $\begin{array}{c}\text { Variabilidad media } \\
(\mathrm{mm})\end{array}$ & $\begin{array}{c}\text { Desviación estándar } \\
(\mathrm{mm})\end{array}$ \\
\hline Tablillas & Longitud & 240,0 & 6 & 8 \\
$(240 \times 12 \times 3 \mathrm{~cm})$ & Ancho 1 & 11,4 & 2 & 3 \\
& Ancho centro & 11,4 & 1 & 1 \\
& Ancho 2 & 11,4 & 2 & 5 \\
& Espesor1 & 3,0 & 1 & 2 \\
& Espesor centro & 3,0 & 1 & 2 \\
& Espesor 2 & 3,0 & 1 & 2 \\
\hline Tablas & Longitud & 300,2 & 9 & 8 \\
$300 \times 20 \times 2 \mathrm{~cm})$ & Ancho 1 & 19,7 & 2 & 8 \\
& Ancho centro & 19,6 & 2 & 3 \\
& Ancho 2 & 19,6 & 2 & 2 \\
& Espesor 1 & 2,3 & 2 & 2 \\
& Espesor centro & 2,2 & 2 & 2 \\
& Espesor 2 & 2,2 & 2 & 2 \\
\hline
\end{tabular}

Cuadro 2. Prueba de T análisis de grupos* de los defectos en tablas y tablillas de Eucalyptus saligna.

T-Test analysis groups* of defects in tables and splints of Eucalyptus saligna.

\begin{tabular}{|c|c|c|c|c|c|c|c|}
\hline \multirow{2}{*}{ Defecto } & \multirow{2}{*}{\multicolumn{2}{|c|}{ Parámetro }} & \multirow{2}{*}{ Valor } & \multirow{2}{*}{ Sesgo } & \multirow{2}{*}{$\begin{array}{c}\text { Desviación } \\
\text { estándar (sd) }\end{array}$} & \multicolumn{2}{|c|}{ Intervalo de confianza al $95 \%$} \\
\hline & & & & & & Inferior & Superior \\
\hline \multirow{8}{*}{ Arqueadura } & \multirow{4}{*}{1} & $\mathrm{~N}$ & 300 & & & & \\
\hline & & Media & 0,96 & $-0,0007$ & 0,0265 & 0,9095 & 1,0173 \\
\hline & & Desviación estándar. & 0,46 & $-0,0024$ & 0,0285 & 0,3981 & 0,5129 \\
\hline & & Error típico de la media & 0,03 & & & & \\
\hline & \multirow{4}{*}{2} & $\mathrm{~N}$ & 300 & & & & \\
\hline & & Media & 0,65 & $-0,0015$ & 0,0279 & 0,5918 & 0,7043 \\
\hline & & Desviación estándar. & 0,49 & $-0,0025$ & 0,024 & 0,4416 & 0,5409 \\
\hline & & Error típico de la media & 0,03 & & & & \\
\hline \multirow{8}{*}{ Encorvado } & \multirow{4}{*}{1} & $\mathrm{~N}$ & 300 & & & & \\
\hline & & Media & 1,07 & 0,001 & 0,0281 & 1,0201 & 1,128 \\
\hline & & Desviación estándar & 0,51 & $-0,0009$ & 0,021 & 0,4615 & 0,5462 \\
\hline & & Error típico de la media & 0,03 & & & & \\
\hline & \multirow{4}{*}{2} & $\mathrm{~N}$ & 300 & & & & \\
\hline & & Media & 1,72 & 0,0005 & 0,0391 & 1,6438 & 1,7965 \\
\hline & & Desviación estándar & 0,69 & $-0,0013$ & 0,0269 & 0,6365 & 0,7412 \\
\hline & & Error típico de la media & 0,04 & & & & \\
\hline
\end{tabular}




\begin{tabular}{|c|c|c|c|c|c|c|c|}
\hline \multirow{8}{*}{ Torcedura } & \multirow{4}{*}{1} & $\mathrm{~N}$ & 300 & & & & \\
\hline & & Media & 1,03 & $-0,0019$ & 0,0285 & 0,9733 & 1,0842 \\
\hline & & Desviación estándar & 0,48 & $-0,0034$ & 0,0278 & 0,425 & 0,5339 \\
\hline & & Error típico de la media & 0,03 & & & & \\
\hline & \multirow{4}{*}{2} & $\mathrm{~N}$ & 300 & & & & \\
\hline & & Media & 2,24 & 0,0035 & 0,0503 & 2,147 & 2,3387 \\
\hline & & Desviación estándar & 0,85 & $-0,0004$ & 0,0323 & 0,7857 & 0,9181 \\
\hline & & Error típico de la media & 0,049 & & & & \\
\hline \multirow{8}{*}{ Acanaladura } & \multirow{5}{*}{1} & $\mathrm{~N}$ & 300 & & & & \\
\hline & & Media & 0,18 & 0 & 0,0133 & 0,1555 & 0,2071 \\
\hline & & Desviación estándar & 0,24 & $-0,0115$ & 0,0679 & 0,1286 & 0,3608 \\
\hline & & Error típico de la media & 0,01 & & & & \\
\hline & & $\mathrm{N}$ & 300 & & & & \\
\hline & \multirow{3}{*}{2} & Media & 0,29 & 0,0002 & 0,0085 & 0,2749 & 0,3082 \\
\hline & & Desviación estándar & 0,15 & $-0,0028$ & 0,0212 & 0,1117 & 0,1884 \\
\hline & & Error típico de la media & 0,01 & & & & \\
\hline
\end{tabular}

*. Análisis de grupos: 1.=Tablillas y 2.=Tablas (SPSS versión 19).

Cuadro 3. Defectos evaluados en piezas aserradas de $240 \times 12$ × $3 \mathrm{~cm}$ (tablillas). Defects sawn evaluated in $240 \times 12 \times 3 \mathrm{~cm}$ (splints).

\begin{tabular}{|c|c|c|c|c|c|c|c|}
\hline Defectos & $\begin{array}{c}\text { Cóncavo } \\
\mathrm{cm}\end{array}$ & $\begin{array}{c}\text { Convexo } \\
\mathrm{cm}\end{array}$ & $\begin{array}{c}\text { Largo } \\
\mathrm{cm}\end{array}$ & $\begin{array}{l}\text { Acho } \\
\mathrm{cm}\end{array}$ & $\begin{array}{c}\text { Espesor } \\
\mathrm{cm}\end{array}$ & $\begin{array}{c}\text { Volumen } \\
\mathrm{cm}^{3}\end{array}$ & $\begin{array}{c}\text { Pérdida } \\
\%\end{array}$ \\
\hline Rajaduras & 32,3 & 27,2 & 59,5 & 11,4 & 3,0 & $2.019,5$ & 24,8 \\
\hline Encorvadura & 1,1 & 0,8 & 240,0 & 1,8 & 3,0 & $1.303,4$ & 16,0 \\
\hline Arqueadura & 1,0 & 0,7 & 240,0 & 11,4 & 1,7 & $4.586,9$ & 56,4 \\
\hline Torcedura & 1,0 & --- & 240,0 & 11,4 & 1,0 & $2.829,7$ & 34,8 \\
\hline Acanaladura & 0,2 & 0,1 & 240,0 & 11,4 & 0,3 & 748,0 & 9,2 \\
\hline Rendimiento limpio & & & 180,4 & 9,6 & 1,3 & $2.243,7$ & 27,6 \\
\hline Aserrado Inicial & 240,0 & 239,9 & 240,0 & 11,5 & 3,0 & $8.139,8$ & 100,0 \\
\hline
\end{tabular}

Cuadro 4. Defectos evaluados en piezas aserradas de $300 \times 20 \times 2 \mathrm{~cm}$ (tablas). Defects sawn evaluated in $300 \times 20 \times 2 \mathrm{~cm}$.

\begin{tabular}{lccccrrrr}
\hline \multicolumn{1}{c}{ Defectos } & $\begin{array}{c}\text { Cóncavo } \\
\mathrm{cm}\end{array}$ & $\begin{array}{c}\text { Convexo } \\
\mathrm{cm}\end{array}$ & $\begin{array}{c}\text { Largo } \\
\mathrm{cm}\end{array}$ & $\begin{array}{c}\text { Ancho } \\
\mathrm{cm}\end{array}$ & $\begin{array}{c}\text { Espesor } \\
\mathrm{cm}\end{array}$ & \multicolumn{2}{c}{$\begin{array}{c}\text { Volumen } \\
\mathrm{cm}^{3}\end{array}$} & $\begin{array}{c}\text { Pérdida } \\
\%\end{array}$ \\
\hline Rajaduras & 32,0 & 31,7 & 63,7 & 19,6 & 2,3 & $2.807,4$ & 21,2 \\
Encorvadura & 0,7 & 0,7 & 300,2 & 1,3 & 2,3 & 905,2 & 6,8 \\
Arqueadura & 1,7 & 1,6 & 300,2 & 19,6 & 3,3 & $19.243,0$ & 145,3 & 93,6 \\
Torcedura & 2,2 & --- & 300,2 & 19,6 & 2,2 & $13.181,7$ & $5.708,2$ & 43,1 \\
Acanaladura & 0,3 & 0,7 & 300,2 & 19,6 & 1,0 & $-1,0$ & $-4.406,3$ & $-33,3$ \\
\hline Rendimiento limpio & & & 236,6 & 18,3 & 19,6 & 2,3 & $13.240,6$ & 100,0 \\
\hline Aserrado Inicial & 300,3 & 300,2 & 300,2 & 19,6 & & & & \\
\hline
\end{tabular}




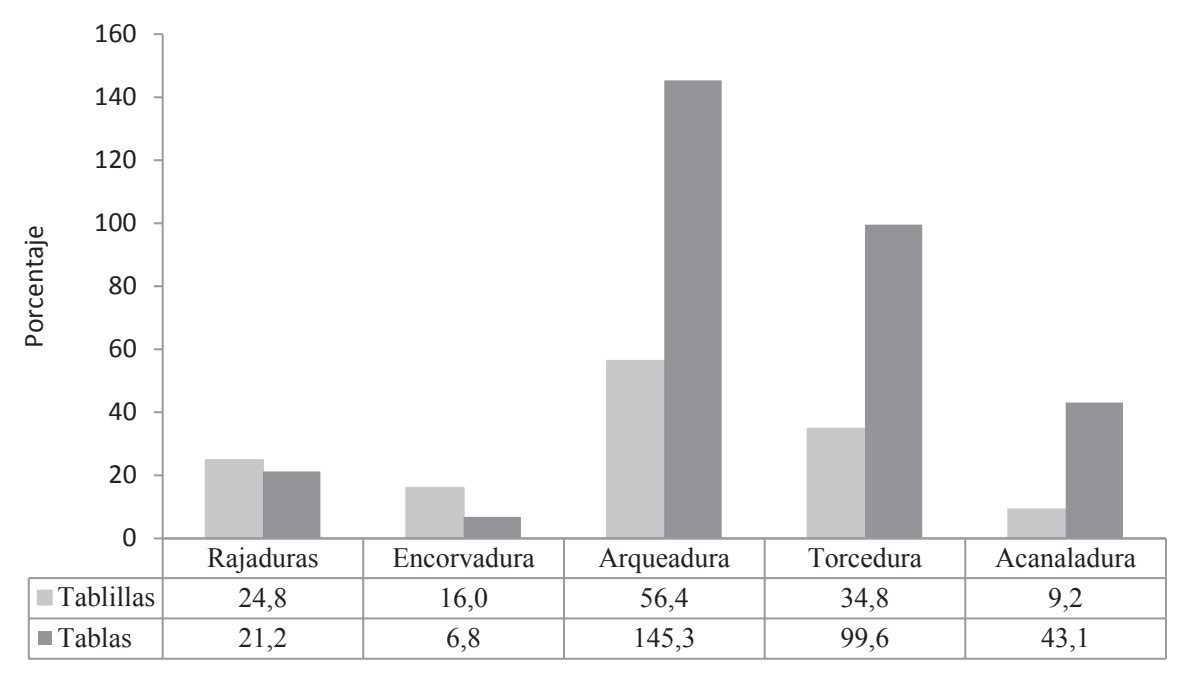

Figura 1. Pérdidas individualizadas por defectos en tablas y tablillas de E. saligna.

Losses individualized by defects in boards and splints E. saligna.

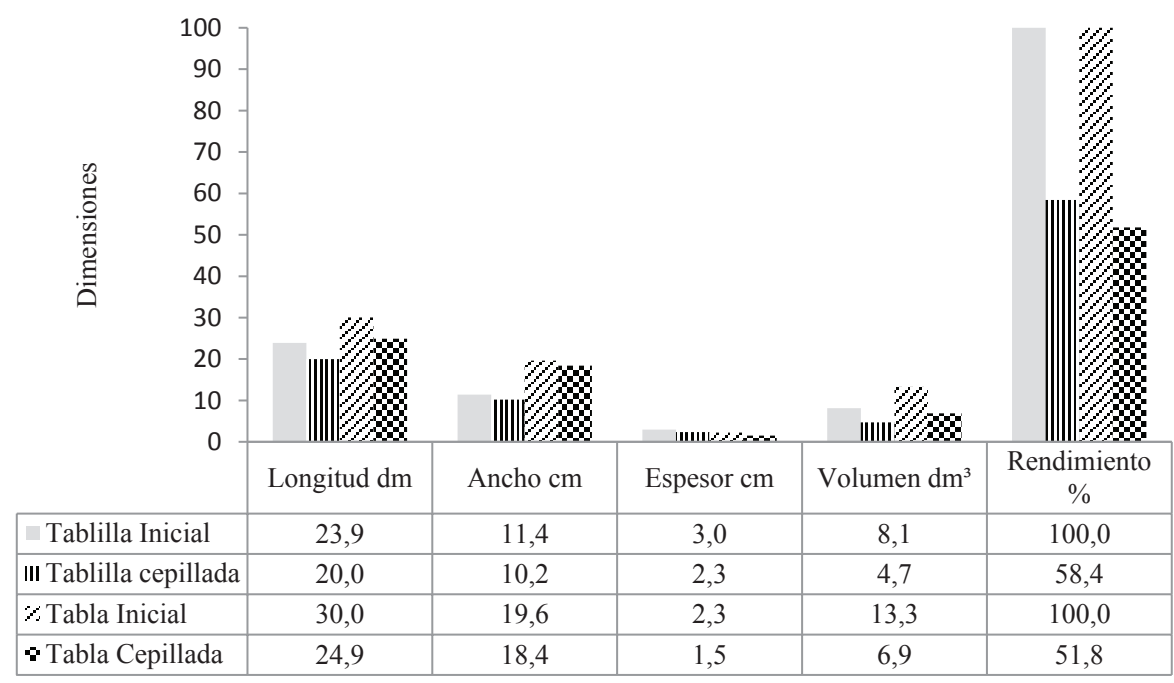

Figura 2. Rendimiento de madera de E. saligna sometida a proceso de eliminación de defectos.

Wood yield E. saligna subjected to defect removal process.

\section{DISCUSIÓN}

Las maderas, por su ultraestructura y composición química, como también por sus propiedades físicas y mecánicas, varían significativamente entre especies, entre árboles de una misma especie y entre partes de un mismo árbol (Da Silva Oliveira y Castro 2003). Tomazello (1985) reportó una variación en la densidad de la madera en sentido radial médula-corteza lo que está relacionado a una mejor calidad de la madera.

La variación dimensional de la madera es una de las causas que dificultan la comercialización y por consecuencia la baja competitividad de la industria maderera, en una experiencia de más de 500 piezas de $28 \mathrm{~mm}$ de espesor Nájera et al. (2006) determinaron que solo un $8 \%$ en aserrado mixto, $33 \%$ en piezas tangenciales y un $38 \%$ en piezas radiales cumplían el espesor especificado. En el ensayo la variación obtenida en espesor es mayor en tablas debido a que estas fueron aserradas con motosierra, en cambio las tablillas fueron aserradas con sierra cinta. Pese a que la variación longitudinal es la más alta, 6 y $9 \mathrm{~mm}$ para tablillas y tablas respectivamente, al presentarse en extremos de la pieza y afectar en el ancho al hacer escuadría, no representa un valor significativo. Sin embargo, la longitud de la pieza influye en los defectos posteriores. La desviación estándar en el ancho de las piezas es similar 
en los dos tipos de piezas y es afectada por la contracción propia de la madera, por lo que el tipo de aserrado radial o tangencial es una condición que debe tenerse en cuenta.

La variación del espesor acentúa los defectos en el proceso de secado, en la práctica afecta a todo el lote de piezas porque tiene que reducirse al menor espesor (figura 1), el limite más bajo de $1,5 \mathrm{~cm}$ de espesor al que hay que reducir en el proceso de cepillado (figura 2), por lo que un nuevo ensayo deberá eliminar mediante un cepillado esta variabilidad dimensional de aserrado, para poder apreciar de manera directa los defectos de secado.

En el caso del defecto de encorvadura afectó a lo largo de la dimensión longitudinal o en los cantos, igualmente, al evaluarse en el lado cóncavo de la pieza, también se produce defecto en el lado convexo y la corrección o eliminación del defecto duplica la pérdida ocasionada al escuadrar la madera.

Luego del análisis de defectos se puede apreciar que las rajaduras, presentes en los dos extremos de las piezas y, en muchos casos, sobrepasa la norma de la JUNAC (1984), que manifiesta se acepta en un solo extremo de la pieza y su longitud no debe sobrepasar el ancho de la pieza. Andrade y Rabelo (1999), al analizar índices de rajaduras, consideran que las rajaduras por sobre el punto de saturación de las fibras están directamente relacionadas a las tensiones de crecimiento. Por tanto, este defecto no es atribuible directamente a secado, pero es uno de los problemas que hay que mejorar, porque es el que mayormente afecta el rendimiento. Para el caso en estudio, un volumen de $24,8 \%$ fue la pérdida directa si se considera solo el defecto de rajaduras al momento de escuadrar la madera, en el que estarían incluidas las rajaduras por tensiones de crecimiento.

Comparado por índice de rajaduras según Taylor (2000), mayor a $10 \%$ califica como fuerte, el porcentaje dimensional obtenido sobre la longitud de la pieza el valor es de $24,8 \%$ coincide con la pérdida de volumen y su calificación de defectos de rajadura sería fuerte. Esta pérdida por rajaduras, señala Sepliarsky (2002), es muy importante en especies similares como $E$. grandis y considera que se puede corregir minimizando el tiempo entre el apeo del árbol y su procesado, así como sellar los extremos para evitar una pérdida rápida del agua. La retirada de rajaduras en tablas de 3,5 m de longitud, Del Menezzi y Sousa (2001) obtuvieron una reducción del $19 \%$, en E. cloeziana, reconociendo al igual que Lobào et al. (2004) que en Brasil las especies más usadas son: E. grandis $55 \%$, E. saligna $17 \%$ y E. urophylla $9 \%$, quienes determinaron una amplia variabilidad en cuanto a densidad del E. grandis.

La encorvadura defecto que se presenta en el canto, también es una eliminación directa del lado cóncavo por donde se empieza el canteo y luego su lado convexo para unificar la dimensión de la pieza. El $16 \%$ de volumen calculado para este defecto es una pérdida que puede corregirse utilizando piezas de menores longitudes.

Los defectos de arqueadura, torcedura y acanaladura tradicionalmente o por concepto se han evaluado en la cara cóncava de la pieza, pero se presentan y proyectan en el lado convexo de la pieza y por tanto estos defectos al ser corregidos mediante cepillado duplican la pérdida de madera. Por ventaja estos no son aditivos en toda su magnitud y el defecto de mayor magnitud enmascara a los de menor valor, por ejemplo, si hay arqueadura al corregirse o cepillarse, en parte ya se elimina o se corrige si hay acanaladura o a la inversa al corregirse una acanaladura mayor se corrige una arqueadura leve. El cálculo del defecto de arqueadura alcanzó un $56 \%$; sin embargo, por la flexibilidad de la madera, al ser cepillada no se corrige totalmente, puede quedar parte en la pieza y se corrige en el armado o uso de la pieza, por tanto no suman independientemente; pero sí afecta en el volumen de pérdida teórica, que es la razón por la que se obtuvo un volumen negativo. Sánchez (2004) recomienda corte tangencial el cual no produce arqueadura difícil de corregir, produce más combado o acanalado que es corregible.

En la torcedura se cuantificó una pérdida en volumen del $34,7 \%$ es un defecto no aceptable, dependiendo de su dimensión, si no es el mayor defecto este puede corregirse en el proceso de cepillado, al igual que la acanaladura que por lo general se corrige en un cepillado o previo sometimiento de la madera en agua y luego secado bajo presión o carga.

Como la corrección de defectos implica una disminución ya sea en el canto, en la cara o en toda la pieza, hay que tener presente esa afectación, en el caso del análisis, considerando las tres mayores dimensiones en largo, ancho y espesor, las pérdidas llegan a 97,2 \%, si se elimina directamente en forma independiente. Por ventaja, propiedades de la madera como la flexibilidad, hacen que en la práctica parte del defecto se mantenga en la pieza cepillada. Además como señala Atencia (1982), la aplicación de tecnologías apropiadas al proceso de transformación puede lograr resultados muy alentadores como los alcanzados en un ensayo de tan solo 3,3\% de rajaduras en extremos y $5,1 \%$ de alabeos en tablones de eucalipto.

El rendimiento teórico de madera limpia, obtenido para tablillas mediante [2], es decir, madera libre de defectos alcanzó un $27,7 \%$. En el caso de tablas, si los defectos se eliminaran de forma directa faltaría dimensiones, por tanto, se estima un volumen negativo de $33 \%$, situación que no es real. No obstante Touza y Pedras (2002) reportan en estudios de eucalipto que en el conjunto del proceso de secado, las pérdidas por defectos y mermas suelen estimarse en torno al $30 \%$ del volumen inicial de madera.

En el ensayo de cepillado de muestras (figura 2), al comparar volumen de madera escuadrada y la madera aserrada, se obtuvo un $58,4 \%$ de rendimiento para tablillas y $51,8 \%$ de rendimiento para tablas, corroborando que los defectos no son aditivos y que el menor dimensionamiento de la madera en tablillas logró un mejor aprovechamiento; se demostró así la hipótesis que hay una importante pérdida de madera que no solo significa volumen, sino que al presentarse luego de varios procesos, es pérdida también 
en trabajo, en operación de maquinaria, afectando costos y rendimientos, lo que justifica mayores esfuerzos investigativos, como sugiere Nahuz (2004), la necesidad de apoyo para investigación en calidad de la madera y su procesamiento para productos provenientes del bosque plantado.

\section{CONCLUSIONES}

La variación dimensional en espesor de las piezas evaluadas con desviación estándar de $1 \mathrm{~mm}$ en tablillas y $2 \mathrm{~mm}$ para tablas, influye mayormente en las pérdidas por cepillado de la madera y demuestra que la variación dimensional es un factor que debe disminuirse a fin de evitar estas pérdidas.

Los defectos de acanaladura y arqueaduras, afectan mayormente durante el cepillado y su magnitud, como es lógico, por su determinación es proporcional a la dimensión de la pieza, razón por lo que las pérdidas son menores en tablillas; es decir, parte de los defectos se pueden corregir utilizando piezas de menores dimensiones, toda vez que la corrección implica doble pérdida.

El rendimiento de madera limpia luego del cepillado para tablillas de $58,4 \%$ y $51,8 \%$ de rendimiento para tablas significa que las piezas de menor dimensión en la madera de E. saligna obtienen un mejor aprovechamiento. Además, permite cuantificar los defectos demostrando que hay una pérdida importante de madera de E. saligna, que debe ser corregida durante el proceso de aserrado y secado a fin evitar pérdidas no solo de madera, sino de tiempo, trabajo y energía o esfuerzo de maquinaria.

\section{REFERENCIAS}

Atencia ME. 1982. Secado de Eucalyptus grandis de $40 \mathrm{~mm}$ de espesor para fabricación de muebles. Revista Asora 13(78):68-72. Consultado 13 ago. 2013. Disponible en: http://www.sidalc.net/

Calvo F, AD Cotrina, AG Cuffré, JC Piter, PM Stefani, EA Torrán. 2006. Variación radial y axial del inchamiento del factor anisotrópico y de la densidad en el Eucalyptus grandis de Argentina. Maderas: Ciencia y Tecnología 8(3): 159-168.

CATIE (Centro Agronómico Tropical de Investigación y Enseñanza, CR). 1991. Eucalyptus saligna Smith especie de árbol de multipropósito en América Central. Informe Técnico $\mathrm{N}^{\circ}$ 184. Turrialba, Costa Rica. CATIE. 71 p.

Da Silva Oliveira JT, J de Castro Silva. 2003. Variação radial da retratibilidade e densidade básica da madeira de Eucalyptus saligna Sm. Revista Árvore 27(3):381-385.

De Andrade MJ, MA Rabelo. 1999. Estudo da influência do espaçamento de plantio de Eucalyptus saligna Smith nos indices de rachamento após o desdobro e após a secagem. Sientia Forestalis (55):107-116.

DeBell D, C Keves, B Gartner. 2001. Wood density of Eucalyptus saligna grown in Hawaiian plantations: effects of silvicultural practices and relation to growth rate. Australian Forest 64(2): 106-110. DOI: 10.1080/00049158.2001.10676173

De Freitas Amparado K. 2008. Qualidade da madeira serrada e dos painéis colados lateralmente obtidos de un plantio de
Eucalyptus saligna Smith Visando o Segmento Movileiro. Mestre em Ciências no curso de Pós-Graduacao em Ciências Ambientais e Florestais. Río de Janeiro, Brasil. Instituto de Florestas. Universida de Federal Rural Do Río de Janeiro. 59 p.

De Freitas Amparado, K Monteiro de Carvalho, A Aparecida García, R De Figueredo, JV Latorraca. 2008. Caracterização do rendimento em madeira serrada de Eucalyptus saligna Smith nas condições verde e seca. Revista Forestal Venezolana 52(1): 71-76.

Del Menezzi CHS, MR Souza. 2001. Madera de Eucalyptus cloeziana F. Muell.: características de aserrío y secado. In Congreso Forestal Español. Consultado sep. 2015. Disponible en: http://secforestales.org/publicaciones/index. php/congresos/article/download/

JUNAC (Junta del Acuerdo de Cartagena, PE). 1984. Manual de clasificación visual de madera estructural. Lima-Perú. PADAT-REFORT. 76 p.

Lobão MS, RMD Lúcia, MSS Moreira, A Gomes. 2004. Caracterização das propiedades físico-mecânicas da madeira de eucalipto com diferentes densidades. Revista Árvore 28(6): 889-894.

López A, L Parisi 2002. Tensiones de crecimiento del estrato dominante de algunos orígenes y procedencias de Eucalyptus grandis a los 17 años de edad en 2 sitios del oeste de la provincia de Corrientes, Argentina. In XVII Jornadas Forestales de Entre Ríos, 24-25 octubre 2002. Consultado 20 sep. 2013. Disponible en http://www.orton.catie.ac.cr.

López N. 2001. Efecto de la dirección de corte de aserrado en el secado convencional de madera de eucalipto colorado ( $E u$ calyptus saligna). Tesis Magíster en Ciencias Forestales . Santiago, Chile. Universidad de Chile. 97 p.

Martin ISSN 1667-9253s D. 2009. Avaliacao do uso de estufa solar para secagem de madeira serrada de eucalipto. Disertacao de Mestrado. Santa Maria, Brasil. Programa de Posgraduacao em Engenheria Florestal. Universidade Santa María. 113 p.

Nahuz M. 2004. Experiencia en Brasil en productos sólidos de eucalipto. In XIX Jornadas Forestales de Entre Ríos, Concordia, Argentina, octubre de 2004. Disponible en CD. ISSN 1667-9253.

Nájera JA, IR Reta, J Méndez González, JJ Graciano Luna, Rosas García, FJ Hernández. 2006. Evaluación de tres sistemas de asierre en Quercus sideroxyla Humb y Bompl. de El Salto, Durango. Ra Ximhai 2(2): 497-513.

Pérez P, Ananías R, Hernández G. 2007. Estudio experimental del secado de renovales de canelo Drimys winteri. Maderas. Ciencia y Tecnología 9(1): 59-70

Sánchez Acosta M. 2006. Productos sólidos a partir de madera de eucaliptos cultivados. Concordia, Entre Ríos, Argentina. INTA (Instituto Nacional de Tecnología Agropecuaria), Estación Experimental Concordia. Boletín del CIDEU 2: 103-117.

Sandoval-Torres S. 2009. Modelación matemática del secado convencional de madera. Madera y Bosques 15(1): 75-89.

Sepliarsky F. 2002. Producción y mercado de madera de Eucalyptus grandis. In XVII Jornadas Forestales de Entre Ríos. Córdova-Argentina. 9 p. Consultado sep. 2015. Disponible en http://exploredoc.com/doc/18555/raleotapebicu\%C3\%A1-archivo---categor\%C3\%ADas

Severo ETD. 2000. Qualidade da secagem de madeira serrada de 
Eucalyptus dunnii. Ciência Florestal 10(1): 109-124.

Skolmen R. 1986. Perfomance of Australian provenances of Eucalyptus grandis and Eucalyptus saligna in Hawai. Berkeley, USA. Forest Servive, US Departament of Agriculture. 8 p.

Tomazello M. 1985. Variazao radial da densidad de basica e da estructura anatomica da madeira do E. saligna e E. grandis. IPEF 29: 37-45.

Touza M, F Pedras. 2002. Una propuesta industrial de secado de madera de eucalipto blanco (Eucalyptus globulus) de Galicia. Productos y Tecnología. Revista CIS-Madera 8: 28-36.

Recibido: 16.11 .13

Aceptado: 28.12.15 